\title{
A 2 : 1 Coupling Reaction of Arynes with Aldehydes via o-Quinone Methides: Straightforward Synthesis of 9-Arylxanthenes
}

\author{
Hiroto Yoshida,* Masahiko Watanabe, Hiroyuki Fukushima, Joji Ohshita and \\ Atsutaka Kunai*
Department of Applied Chemistry, Graduate School of Engineering, Hiroshima University, Higashi-Hiroshima 739-8527, Japan.
E-mail:yhiroto@hiroshima-u.ac.jp,akunai@hiroshima-u.ac.jp

\section{Supporting Information}

General Remarks. All manipulations of oxygen- and moisture-sensitive materials were conducted with a standard Schlenk technique under a purified argon atmosphere. Melting points were determined using a Yanaco micro melting point apparatus. Nuclear magnetic resonance spectra were taken on a JEOL EX-270 $\left({ }^{1} \mathrm{H}, 270 \mathrm{MHz} ;{ }^{13} \mathrm{C}, 67.8 \mathrm{MHz}\right)$ spectrometer or a JEOL Lambda-400 $\left({ }^{1} \mathrm{H}, 400 \mathrm{MHz} ;{ }^{13} \mathrm{C}, 99.5 \mathrm{MHz}\right)$ spectrometer using residual chloroform $\left({ }^{1} \mathrm{H}\right)$ or $\mathrm{CDCl}_{3}\left({ }^{13} \mathrm{C}\right)$ as an internal standard. High-resolution mass spectra were obtained with a Hitachi M-80B spectrometer or a JEOL JMS-HX110A spectrometer. The preparative recycling gel permeation chromatography was performed with GL Science PU 614 equipped with Shodex GPC H-2001L and -2002L columns (chloroform as an eluent). Column chromatography was carried out using Merck Kieselgel 60. Unless otherwise noted, commercially available reagents were used without purification. 18-Crown-6 was recrystallized from distilled MeCN. KF (spray-dried) was vacuum dried at $100{ }^{\circ} \mathrm{C}$ for $12 \mathrm{~h}$. THF was distilled from sodium/benzophenone ketyl. MeCN was distilled from phosphorus pentoxide.

A ry n Precursors. $\quad 2$-(Trimethylsilyl)phenyl triflate (1a), ${ }^{1} \quad$ 4,5-dimethyl-2(trimethylsilyl)phenyl triflate (1b), ${ }^{2}$ 6-(trimethylsilyl)-5-indanyl triflate (1c), ${ }^{2}$ 3,6-dimethoxy-2(trimethylsilyl)phenyl triflate (1d) ${ }^{2}$ and 3-methoxy-2-(trimethylsilyl)phenyl triflate $(\mathbf{1 e})^{3}$ were prepared according to literature procedures.
Aldehydes.
2-Benzyloxy-1-naphthaldehyde
$(\mathbf{2 a})^{4}$
and
$2,6-$ dibenzyloxybenzaldehyde $(\mathbf{2 e})^{5}$ were synthesized according to literature methods. 3,3'- Diformyl-4,4'-dimethoxydiphenyl ether (2o) was prepared from 3,3'-dibromo-4,4'- dimethoxydiphenyl ether in a similar manner as reported. ${ }^{6}$ 3,3'-Dibromo-4,4'- dimethoxydiphenyl ether was synthesized by the methylation of 3,3'-dibromo-4,4'- dihydroxydiphenyl ether ${ }^{7}$ with methyl iodide (8 eq) and $\mathrm{K}_{2} \mathrm{CO}_{3}(8 \mathrm{eq})$ in refluxing acetone. 
Reaction of Arynes with Aldehydes. A GeneralProcedure. To a THF solution (1.0 $\mathrm{mL})$ of an aryne precursor $(0.45 \mathrm{mmol})$, an aldehyde $(0.15 \mathrm{mmol})$ and 18 -crown-6 $(0.238 \mathrm{~g}$, $0.90 \mathrm{mmol})$ was added $\mathrm{KF}(0.052 \mathrm{~g}, 0.90 \mathrm{mmol})$, and the resulting mixture was stirred at $0{ }^{\circ} \mathrm{C}$. After the time specified in Table 1 or Scheme 2, the mixture was diluted with ethyl acetate, filtered through a Celite plug, and concentrated. Silicagel column chromatography (hexane/ethyl acetate as an eluent) followed by gel permeation chromatography (chloroform as an eluent) gave the corresponding product.

9-(2-Benzyloxy-1-naphthyl)xanthene (3aa). (A $65: 35$ mixture of two conformers) Isolated in $70 \%$ yield as a white solid: m.p. $167-168{ }^{\circ} \mathrm{C} ;{ }^{1} \mathrm{H}$ NMR $\left(\mathrm{CDCl}_{3}\right) \delta 4.74$ (s, minor, $\mathrm{CH}_{2} \mathrm{Ph}$ ), 5.37 (s, major, $\left.-\mathrm{CH}_{2} \mathrm{Ph}\right), 6.37$ (s, minor, methine $H$ ), 6.65 (d, $J=7.5 \mathrm{~Hz}$ ), 6.69-6.88 (m), $6.95(\mathrm{~d}, J=8.0 \mathrm{~Hz}), 7.06(\mathrm{t}, J=7.0 \mathrm{~Hz}), 7.09-7.25(\mathrm{~m}), 7.29-7.41(\mathrm{~m}), 7.43-7.54(\mathrm{~m}), 7.56$ $(\mathrm{d}, J=8.7 \mathrm{~Hz}), 7.65(\mathrm{t}, J=7.5 \mathrm{~Hz}), 7.75(\mathrm{~d}, J=8.7 \mathrm{~Hz}), 7.83-7.91(\mathrm{~m}), 8.46(\mathrm{~d}, J=8.9 \mathrm{~Hz})$; ${ }^{13} \mathrm{C} \mathrm{NMR}\left(\mathrm{CDCl}_{3}\right) \delta 33.4,34.4,70.0,72.0,114.7,115.3,115.8,116.1,122.0,122.3,123.1$, $123.3,124.1,124.7,125.7,125.9,126.8,127.1,127.3,127.46,127.52,127.58,127.62,128.0$, 128.1, 128.4, 128.5, 128.6, 128.7, 128.77, 128.82, 129.0, 129.7, 130.7, 131.7, 133.5, 136.8, 137.0, 150.9, 151.3, 154.1, 154.9; Anal. Calcd for $\mathrm{C}_{30} \mathrm{H}_{22} \mathrm{O}_{2}$ C, 86.93; H, 5.35. Found: C, $86.63 ; \mathrm{H}, 5.33$.

\section{${ }^{1}$ H NMR integration ratio}

$4.74(\mathrm{~s}): 5.37(\mathrm{~s}): 6.37(\mathrm{~s}): 6.65(\mathrm{~d}): 6.69-6.88(\mathrm{~m}): 6.95(\mathrm{~d}): 7.06(\mathrm{t}): 7.09-7.25(\mathrm{~m}): 7.29$ $7.41(\mathrm{~m}): 7.43-7.54(\mathrm{~m}): 7.56(\mathrm{~d}): 7.65(\mathrm{t}): 7.75(\mathrm{~d}): 7.83-7.91(\mathrm{~m}): 8.46(\mathrm{~d})=2.11: 3.94:$ $1.05: 3.89: 12.34: 2.41: 2.03: 16.15: 6.05: 6.92: 2.16: 1.18: 3.05: 3.06: 1.00$.

9-(2-Methoxy-1-naphthyl)xanthene (3ab). (A $65: 35$ mixture of two conformers) Isolated in $66 \%$ yield as a white solid: m.p. $219-221{ }^{\circ} \mathrm{C} ;{ }^{1} \mathrm{H}$ NMR $\left(\mathrm{CDCl}_{3}\right) \delta 3.32$ (s, minor, - $\mathrm{OCH}_{3}$ ), 4.06 (s, major, $-\mathrm{OCH}_{3}$ ), 6.29 (s, minor, methine $H$ ), 6.64-6.67 (m), 6.77-6.83 (m), 7.02$7.19(\mathrm{~m}), 7.43-7.48(\mathrm{~m}), 7.52$ (d, $J=4.1 \mathrm{~Hz}), 7.65(\mathrm{t}, J=8.0 \mathrm{~Hz}), 7.75(\mathrm{~d}, J=8.2 \mathrm{~Hz}), 7.80(\mathrm{~d}, J$ $=8.9 \mathrm{~Hz}), 7.88(\mathrm{~d}, J=8.9 \mathrm{~Hz}), 8.46(\mathrm{~d}, J=8.7 \mathrm{~Hz}) ;{ }^{13} \mathrm{C} \mathrm{NMR}\left(\mathrm{CDCl}_{3}\right) \delta 33.2,34.4,56.6$, 57.2, 113.0, 115.6, 116.1, 116.2, 122.2, 122.3, 123.0, 123.2, 123.5, 123.6, 124.2, 124.8, 125.6, $125.9,127.3,127.4,127.5,128.4,128.49,128.52$, 128.8, 129.1, 129.2, 129.8, 130.4, 131.7, 133.2, 151.0, 151.2, 155.7, 156.0; HRMS Calcd for $\mathrm{C}_{24} \mathrm{H}_{18} \mathrm{O}_{2}: \mathrm{M}^{+}, 338.1307$. Found: $\mathrm{m} / \mathrm{z}$ 338.1313 .

\section{${ }^{1}$ H NMR integration ratio}

3.32 (s) : 4.06 (s) : 6.29 (s) : 6.64-6.67 (m) : 6.77-6.83 (m) : 7.02-7.19 (m) : 7.43-7.48 (m) : 7.52 (d) $: 7.65(\mathrm{t}): 7.75$ (d) $: 7.80$ (d) $: 7.88$ (d) $: 8.46(\mathrm{~d})=3.00: 5.68: 0.96: 5.61: 8.08:$ $16.46: 2.90: 2.02: 1.09: 1.77: 1.17: 2.95: 0.97$. 
9-(1-Naphthyl)xanthene (3ac). Isolated in $17 \%$ yield as a white solid: m.p. $159-161{ }^{\circ} \mathrm{C}$; ${ }^{1} \mathrm{H} \mathrm{NMR}\left(\mathrm{CDCl}_{3}\right) \delta 6.02(\mathrm{~s}, 1 \mathrm{H}), 6.82-6.90(\mathrm{~m}, 4 \mathrm{H}), 7.14-7.22(\mathrm{~m}, 4 \mathrm{H}), 7.35-7.46(\mathrm{~m}, 4 \mathrm{H})$, $7.79(\mathrm{~d}, J=8.0 \mathrm{~Hz}, 1 \mathrm{H}), 7.86(\mathrm{~d}, J=8.6 \mathrm{~Hz}, 1 \mathrm{H}), 8.08(\mathrm{~s}, 1 \mathrm{H}) ;{ }^{13} \mathrm{C} \mathrm{NMR}\left(\mathrm{CDCl}_{3}\right) \delta 29.7$, 116.4, 123.1, 123.6, 124.5, 124.8, 125.5, 125.6, 126.1, 127.9, 128.5, 129.0, 129.1, 129.2, 131.1, 134.5, 150.7; HRMS Calcd for $\mathrm{C}_{23} \mathrm{H}_{16} \mathrm{O}: \mathrm{M}^{+}, 308.1201$. Found: $m / z$ 308.1235.

9-(2,6-Dimethoxyphenyl)xanthene (3ad). Isolated in $61 \%$ yield as a white solid: m.p. 158-159 ${ }^{\circ} \mathrm{C}$; ${ }^{1} \mathrm{H}$ NMR $\left(\mathrm{CDCl}_{3}\right) \delta 3.33$ (brs, $3 \mathrm{H}$ ), 3.96 (brs, $3 \mathrm{H}$ ), 6.07 (s, $1 \mathrm{H}$ ), 6.55 (brs, 2 $\mathrm{H}), 6.87(\mathrm{t}, J=7.4 \mathrm{~Hz}, 2 \mathrm{H}), 6.96(\mathrm{~d}, J=7.0 \mathrm{~Hz}, 2 \mathrm{H}), 7.03(\mathrm{~d}, J=8.2 \mathrm{~Hz}, 2 \mathrm{H}), 7.11-7.20$ (m, 3 $\mathrm{H}) ;{ }^{13} \mathrm{C} \mathrm{NMR}\left(\mathrm{CDCl}_{3}\right) \delta 32.0,55.9,115.4,122.3,123.6,124.3,126.9,128.1,128.6,151.5$; HRMS Calcd for $\mathrm{C}_{21} \mathrm{H}_{18} \mathrm{O}_{3}: \mathrm{M}^{+}, 318.1256$. Found: $m / z 318.1179$.

9-(2,6-Dibenzyloxyphenyl)xanthene (3ae). Isolated in 60\% yield as a white solid: m.p. 142-145 ${ }^{\circ} \mathrm{C}$; ${ }^{1} \mathrm{H}$ NMR $\left(\mathrm{CDCl}_{3}\right) \delta 4.67$ (s, $\left.2 \mathrm{H}\right), 5.28$ (s, $2 \mathrm{H}$ ), 6.27 (s, $\left.1 \mathrm{H}\right), 6.40$ (brs, $1 \mathrm{H}$ ), 6.68 (brs, 2 H), 6.75 (brs, $1 \mathrm{H}), 6.84-7.02$ (m, $6 \mathrm{H}), 7.02-7.29$ (m, $6 \mathrm{H})$, 7.29-7.48 (m, $3 \mathrm{H})$, 7.54 (brs, $2 \mathrm{H}) ;{ }^{13} \mathrm{C} \mathrm{NMR}\left(\mathrm{CDCl}_{3}\right) \delta 32.0,69.5,70.7,104.9,106.2,115.6,122.2$ (brs), 123.3, 124.1, 127.1 (brs), 128.1 (brs), 128.6 (brs), 136.7, 137.1, 151.4, 156.5, 157.4; HRMS Calcd for $\mathrm{C}_{26} \mathrm{H}_{19} \mathrm{O}_{3}: \mathrm{M}^{+}-\mathrm{CH}_{2} \mathrm{Ph}, 379.1334$. Found: $m / z$ 379.1331.

9-(2-Methoxyphenyl)xanthene (3af). Isolated in $54 \%$ yield as a white solid: m.p. 143-145 ${ }^{\circ} \mathrm{C} ;{ }^{1} \mathrm{H} \mathrm{NMR}\left(\mathrm{CDCl}_{3}\right) \delta 3.86(\mathrm{~s}, 3 \mathrm{H}), 5.82(\mathrm{~s}, 1 \mathrm{H}), 6.82(\mathrm{t}, J=7.5 \mathrm{~Hz}, 1 \mathrm{H}), 6.90(\mathrm{~d}, J=8.2$ $\mathrm{Hz}, 1 \mathrm{H}), 6.93-6.97(\mathrm{~m}, 3 \mathrm{H}), 7.09-7.21(\mathrm{~m}, 7 \mathrm{H}) ;{ }^{13} \mathrm{C} \mathrm{NMR}\left(\mathrm{CDCl}_{3}\right) \delta 36.9,55.6,111.0$, 116.2, 121.1, 123.0, 124,8, 127.5, 127.7, 129.5, 130.3, 135.3, 151.5, 156.2; HRMS Calcd for $\mathrm{C}_{20} \mathrm{H}_{16} \mathrm{O}_{2}: \mathrm{M}^{+}, 288.1150$. Found: $m / z, 288.1159$.

9-(2,4-Dimethoxyphenyl)xanthene (3ag). Isolated in $53 \%$ yield as a white solid: m.p. 89-91 ${ }^{\circ} \mathrm{C} ;{ }^{1} \mathrm{H}$ NMR $\left(\mathrm{CDCl}_{3}\right) \delta 3.75(\mathrm{~s}, 3 \mathrm{H}), 3.84(\mathrm{~s}, 3 \mathrm{H}), 5.72(\mathrm{~s}, 1 \mathrm{H}), 6.36(\mathrm{dd}, J=8.4,2.4$ $\mathrm{Hz}, 1 \mathrm{H}), 6.48(\mathrm{~d}, J=2.4 \mathrm{~Hz}, 1 \mathrm{H}), 6.84(\mathrm{~d}, J=8.4 \mathrm{~Hz}, 1 \mathrm{H}), 6.96(\mathrm{t}, J=7.5 \mathrm{~Hz}, 2 \mathrm{H}), 7.09-7.21$ $(\mathrm{m}, 6 \mathrm{H}) ;{ }^{13} \mathrm{C} \mathrm{NMR}\left(\mathrm{CDCl}_{3}\right) \delta 36.3,55.2,55.5,98.6,104.7,116.1,123.0,125.0,127.4,128.0$, 129.4, 130.7, 151,4, 157.2, 159.3; HRMS Calcd for $\mathrm{C}_{20} \mathrm{H}_{15} \mathrm{O}_{3}: \mathrm{M}^{+}-\mathrm{Me}, 303.1021$. Found: $\mathrm{m} / \mathrm{z}$ 303.0989 .

9-(2,4,6-Trimethoxyphenyl)xanthene (3ah). Isolated in $44 \%$ yield as a white solid: m.p. 162-163 ${ }^{\circ} \mathrm{C}$; ${ }^{1} \mathrm{H}$ NMR $\left(\mathrm{CDCl}_{3}\right) \delta 3.29$ (brs, $\left.3 \mathrm{H}\right), 3.78$ (s, $\left.3 \mathrm{H}\right), 3.96$ (brs, $\left.3 \mathrm{H}\right), 5.94(\mathrm{~s}, 1 \mathrm{H})$, 6.10 (brs, $2 \mathrm{H}), 6.87$ (t, $J=7.3 \mathrm{~Hz}, 2 \mathrm{H}), 6.96$ (d, $J=7.3 \mathrm{~Hz}, 2 \mathrm{H}$ ), $7.02(\mathrm{~d}, J=8.0 \mathrm{~Hz}, 2 \mathrm{H})$, $7.12(\mathrm{t}, J=7.5 \mathrm{~Hz}, 2 \mathrm{H}) ;{ }^{13} \mathrm{C} \mathrm{NMR}\left(\mathrm{CDCl}_{3}\right) \delta 31.7,55.2,55.9,115.4,116.5,122.2,124.7$, 126.8, 128.56, 151.5, 160.0; HRMS Calcd for $\mathrm{C}_{22} \mathrm{H}_{20} \mathrm{O}_{4}: \mathrm{M}^{+}, 348.1362$. Found: $\mathrm{m} / \mathrm{z}$ 348.1393 . 
9-(4-Methoxyphenyl)xanthene (3ai). Isolated in 42\% yield as a white solid: m.p. 110-112 ${ }^{\circ} \mathrm{C} ;{ }^{1} \mathrm{H} \mathrm{NMR}\left(\mathrm{CDCl}_{3}\right) \delta 3.76(\mathrm{~s}, 3 \mathrm{H}), 5.22(\mathrm{~s}, 1 \mathrm{H}), 6.82(\mathrm{~d}, J=9.1 \mathrm{~Hz}, 2 \mathrm{H}), 7.06(\mathrm{~d}, J=6.8$ $\mathrm{Hz}, 2 \mathrm{H}), 6.98(\mathrm{t}, J=7.0 \mathrm{~Hz}, 2 \mathrm{H}), 7.11-7.14(\mathrm{~m}, 4 \mathrm{H}), 7.21(\mathrm{td}, J=7.6,1.7 \mathrm{~Hz}, 2 \mathrm{H}) ;{ }^{13} \mathrm{C}$ NMR $\left(\mathrm{CDCl}_{3}\right) \delta 43.5,55.2,114.1,116.5,123.2,124.7,127.8,129.3,129.7,138.8,151.0$, 158.3; HRMS Calcd for $\mathrm{C}_{20} \mathrm{H}_{16} \mathrm{O}_{2}: \mathrm{M}^{+}$, 288.1150. Found: $m / z$ 288.1183.

9-(2,4,6-Trimethylphenyl)xanthene (3aj). ${ }^{8}$ Isolated in $58 \%$ yield as a white solid: ${ }^{1} \mathrm{H}$ NMR $\left(\mathrm{CDCl}_{3}\right) \delta 1.65(\mathrm{~s}, 3 \mathrm{H}), 2.31(\mathrm{~s}, 3 \mathrm{H}), 2.55(\mathrm{~s}, 3 \mathrm{H}), 5.90(\mathrm{~s}, 1 \mathrm{H}), 6.70-6.84(\mathrm{~m}, 3 \mathrm{H})$, $6.90(\mathrm{td}, J=7.2,1.0 \mathrm{~Hz}, 2 \mathrm{H}), 7.00(\mathrm{~s}, 1 \mathrm{H}), 7.06(\mathrm{~d}, J=7.6 \mathrm{~Hz}, 2 \mathrm{H}), 7.18(\mathrm{t}, J=7.3 \mathrm{~Hz}, 2 \mathrm{H})$; ${ }^{13} \mathrm{C} \mathrm{NMR}\left(\mathrm{CDCl}_{3}\right) \delta$ 20.6, 20.9, 21.3, 37.1, 116.0, 123.0, 123.7, 127.6, 128.1, 128.6, 131.4, $136.5,137.0,137.4,137.7,150.8$.

9-(2,4-Dimethylphenyl)xanthene (3ak). Isolated in $41 \%$ yield as a white solid: m.p. $113-115{ }^{\circ} \mathrm{C} ;{ }^{1} \mathrm{H}$ NMR $\left(\mathrm{CDCl}_{3}\right) \delta 2.18(\mathrm{~s}, 3 \mathrm{H}), 2.31$ (s, $\left.3 \mathrm{H}\right), 5.52(\mathrm{~s}, 1 \mathrm{H}), 6.88-6.99$ (m, 6 $\mathrm{H})$, 7.05-7.11 (m, $3 \mathrm{H}), 7.19(\mathrm{td}, J=7.4,1.7 \mathrm{~Hz}, 2 \mathrm{H}) ;{ }^{13} \mathrm{C} \mathrm{NMR}\left(\mathrm{CDCl}_{3}\right) \delta$ 20.0, 21.0, 41.0, $116.2,123.1,124.3,127.1,127.7,129.2,131.0,132.0,135.7,136.4,140.6,150.9$; HRMS Calcd for $\mathrm{C}_{21} \mathrm{H}_{18} \mathrm{O}: \mathrm{M}^{+}, 286.1358$. Found: $m / z$ 286.1349.

9-(2,6-Dimethylphenyl)xanthene (3al). ${ }^{8}$ Isolated in $38 \%$ yield as a white solid: ${ }^{1} \mathrm{H}$ NMR $\left(\mathrm{CDCl}_{3}\right) \delta 1.69(\mathrm{~s}, 3 \mathrm{H}), 2.59(\mathrm{~s}, 3 \mathrm{H}), 5.94(\mathrm{~s}, 1 \mathrm{H}), 6.74(\mathrm{~d}, J=7.5 \mathrm{~Hz}, 2 \mathrm{H}), 6.88-6.95$ (m, 3 H) $7.07(\mathrm{~d}, J=8.2 \mathrm{~Hz}, 2 \mathrm{H}), 7.12-7.21(\mathrm{~m}, 4 \mathrm{H}) ;{ }^{13} \mathrm{C} \mathrm{NMR}\left(\mathrm{CDCl}_{3}\right) \delta 21.7,21.3,37.4,116.1$, $123.1,123.5,127.1,127.7,127.9,128.0,130.7,137.2,138.0,140.3,150.9$.

9-Phenylxanthene (3am). Isolated in $22 \%$ yield as a white solid: m.p. $136-137{ }^{\circ} \mathrm{C} ;{ }^{1} \mathrm{H}$ NMR $\left(\mathrm{CDCl}_{3}\right) \delta 5.26(\mathrm{~s}, 1 \mathrm{H}), 6.98(\mathrm{t}, J=7.0 \mathrm{~Hz}, 2 \mathrm{H}), 7.06(\mathrm{~d}, J=7.5 \mathrm{~Hz}, 2 \mathrm{H}), 7.13(\mathrm{~d}, J=$ $8.2 \mathrm{~Hz}, 2 \mathrm{H}), 7.17-7.29(\mathrm{~m}, 7 \mathrm{H}) ;{ }^{13} \mathrm{C} \mathrm{NMR}\left(\mathrm{CDCl}_{3}\right) \delta 44.4,116.5,123.2,124.4,126.6,127.9$, 128.4, 128.7, 129.7, 146.5, 151.0; HRMS Calcd for $\mathrm{C}_{19} \mathrm{H}_{14} \mathrm{O}: \mathrm{M}^{+}, 258.1045$. Found: $\mathrm{m} / \mathrm{z}$ 258.1068 .

9-(2-Benzyloxy-1-naphthyl)-2,3,6,7-tetramethylxanthene (3ba). (A $70: 30$ mixture of two conformers) Isolated in $51 \%$ yield as a green solid; m.p. $219-222{ }^{\circ} \mathrm{C}$; ${ }^{1} \mathrm{H}$ NMR $\left(\mathrm{CDCl}_{3}\right) \delta 1.94(\mathrm{~s}), 2.01$ (s), 2.16-2.27 (m), 4.78 (s, minor, $-\mathrm{CH}_{2} \mathrm{Ph}$ ), 5.39 (s, major, $-\mathrm{CH}_{2} \mathrm{Ph}$ ), 6.24 (s, minor, methine $H$ ), 6.40 (s), 6.56 (s), 6.64 (s), 6.69 (d, $J=7.2 \mathrm{~Hz}), 6.74$ (s), 6.90 (s), 7.02-7.25 (m), 7.29-7.61 (m), 7.62-7.79 (m), 7.79-7.93 (m), 8.49 (d, $J=8.9 \mathrm{~Hz}) ;{ }^{13} \mathrm{C}$ NMR $\left(\mathrm{CDCl}_{3}\right) \delta 18.7,18.8,18.9,19.0,19.1,19.4,19.5,32.6,32.7,33.8,71.9,114.7,114.8,115.6$, $115.7,116.2,116.5,116.6,116.8,116.9,121.1,121.5,122.3,123.3$ (brs), 125.5, 125.7, 125.9 (brs), 126.1, 126.9, 127.0, 127.2, 127.6, 127.7, 127.9, 128.1, 128.2, 128.3, 128.5, 128.6, 128.8, 
$128.9,129.08,129.13,129.2,129.4,130.0,130.65,130.69,132.0,133.6,135.3,135.7,137.1$; HRMS Calcd for $\mathrm{C}_{27} \mathrm{H}_{23} \mathrm{O}_{2}: \mathrm{M}^{+}-\mathrm{CH}_{2} \mathrm{Ph}, 379.1698$. Found: $m / z 379.1690$.

${ }^{1} \mathrm{HNMR}$ integration ratio

$1.94(\mathrm{~s}): 2.01(\mathrm{~s}): 2.16-2.27(\mathrm{~m}): 4.78(\mathrm{~s}): 5.39(\mathrm{~s}): 6.24(\mathrm{~s}): 6.40(\mathrm{~s}): 6.56(\mathrm{~s}): 6.64(\mathrm{~s})$ 6.69 (d) : $6.74(\mathrm{~s}): 6.90(\mathrm{~s}): 7.02-7.25(\mathrm{~m}): 7.29-7.61(\mathrm{~m}): 7.62-7.79(\mathrm{~m}): 7.79-7.93(\mathrm{~m}):$ 8.49 (d) $=13.75: 6.99: 22.25: 2.00: 4.64: 1.01: 4.64: 2.03: 2.20: 2.25: 2.62: 4.77: 9.16:$ $15.55: 6.77: 3.48: 1.00$.

9-(2-Benzyloxy-1-naphthyl)-dicyclopenta[b,i]xanthene (3ca). (A $70: 30$ mixture of two conformers) Isolated in $50 \%$ yield as a dark brown solid: m.p. decomposed; ${ }^{1} \mathrm{H}$ NMR $\left(\mathrm{CDCl}_{3}\right) \delta 1.87-2.21(\mathrm{~m}), 2.47-2.76(\mathrm{~m}), 2.76-3.02(\mathrm{~m}), 4.81\left(\mathrm{~s}\right.$, minor, $\left.-\mathrm{CH}_{2} \mathrm{Ph}\right), 5.37(\mathrm{~s}$, major, $-\mathrm{CH}_{2} \mathrm{Ph}$ ), 6.31 (s, minor, methine $H$ ), $6.45(\mathrm{~s}), 6.61-6.74(\mathrm{~m}), 6.74-6.86(\mathrm{~m}), 6.93-7.03$ (m), 7.03-7.22 (m), 7.28-7.39 (m), 7.39-7.57 (m), 7.57-7.78 (m), 7.78-7.92 (m), $8.48(\mathrm{~d}, J=8.7$ $\mathrm{Hz}) ;{ }^{13} \mathrm{C} \mathrm{NMR}\left(\mathrm{CDCl}_{3}\right) \delta 25.55,25.64,25.8,32.0,32.68,32.72,32.8,33.7,33.8,34.9,69.9$, 72.0, 72.4, 100.5, 111.3, 111.4, 111.5, 111.6, 111.8, 114.85, 114.91, 115.0, 115.6, 115.7, 121.6, $122.1,122.3,123.0,123.3,123.55,123.63,123.9,124.4,125.8,125.9,126.1,126.2,126.8$, $126.9,127.2,127.7,127.8,127.9,128.0,128.3,128.4,128.5,128.6,128.8,129.20,129.24$, 129.4, 130.7, 132.0, 133.6, 137.0, 137.1, 137.6, 138.4, 143.2, 143.5, 149.8, 150.3, 153.9, 154.5; HRMS Calcd for $\mathrm{C}_{29} \mathrm{H}_{23} \mathrm{O}_{2}: \mathrm{M}^{+}-\mathrm{CH}_{2} \mathrm{Ph}, 403.1698$. Found: $m / z 403.1684$.

${ }^{1}$ H NMR integration ratio

$1.87-2.21(\mathrm{~m}): 2.47-2.76(\mathrm{~m}): 2.76-3.02(\mathrm{~m}): 4.81(\mathrm{~s}): 5.37(\mathrm{~s}): 6.31(\mathrm{~s}): 6.45(\mathrm{~s}): 6.61-$ $6.74(\mathrm{~m}): 6.74-6.86(\mathrm{~m}): 6.93-7.03(\mathrm{~m}): 7.03-7.22(\mathrm{~m}): 7.28-7.39(\mathrm{~m}): 7.39-7.57(\mathrm{~m}): 7.57-$ $7.78(\mathrm{~m}): 7.78-7.92(\mathrm{~m}): 8.48(\mathrm{~d})=18.78: 17.86: 20.10: 2.21: 5.37: 1.12: 5.58: 8.21:$ $2.80: 5.73: 12.12: 9.39: 10.10: 8.55: 4.91: 1.00$.

9-(4-Methoxyphenyl)-1,4,5,8-tetramethoxyxanthene (3di). Isolated in $45 \%$ yield as a brown solid: m.p. $142-145{ }^{\circ} \mathrm{C} ;{ }^{1} \mathrm{H}$ NMR $\left(\mathrm{CDCl}_{3}\right) \delta 3.43$ (s, $\left.3 \mathrm{H}\right), 3.79$ (s, $\left.3 \mathrm{H}\right), 3.81(\mathrm{~s}, 3 \mathrm{H})$, $3.83(\mathrm{~s}, 3 \mathrm{H}), 3.88$ (s, $3 \mathrm{H}), 6.81-6.92(\mathrm{~m}, 6 \mathrm{H}), 7.36(\mathrm{~s}, 1 \mathrm{H}), 7.72(\mathrm{~d}, J=8.9 \mathrm{~Hz}, 2 \mathrm{H}) ;{ }^{13} \mathrm{C}$ NMR $\left(\mathrm{CDCl}_{3}\right) \delta 54.2,55.2,56.0,57.0,58.0,84.8,89.7,112.8,114.0,114.3,126.0,126.6$, $127.3,129.8,130.5,131.2,133.9,134.7,149.0,150.5,159.9,190.7$; HRMS Calcd for $\mathrm{C}_{24} \mathrm{H}_{24} \mathrm{O}_{6}: \mathrm{M}^{+}, 408.1573$. Found: $m / z$ 408.1570.

1,5-Dimethoxy-9-(2,4,6-trimethylphenyl)xanthene (3ej). (A $91: 9$ mixture of two conformers $)^{9}$ Isolated in $48 \%$ yield as a white solid; m.p. $125-131{ }^{\circ} \mathrm{C} ;{ }^{1} \mathrm{H}$ NMR $\left(\mathrm{CDCl}_{3}\right)$ $\delta 1.92$ (s, minor, $-\mathrm{CH}_{3}$ ), 1.98 (s, major, $-\mathrm{CH}_{3}$ ), 2.12 (s, major, $-\mathrm{CH}_{3}$ ), 2.15 (s, minor, $-\mathrm{CH}_{3}$ ), 2.30 (s, major, $-\mathrm{CH}_{3}$ ), 3.08 (s, minor, $-\mathrm{OCH}_{3}$ ), 3.24 (s, major, $-\mathrm{OCH}_{3}$ ), 3.72-3.83 (m), 3.86 (s, minor, $\left.-\mathrm{OCH}_{3}\right), 3.92(\mathrm{~s}), 3.97-4.07(\mathrm{~m}), 4.53(\mathrm{dd}, J=6.3,1.7 \mathrm{~Hz}$, major, methine $H), 5.08(\mathrm{dd}, J=6.4$, $1.7 \mathrm{~Hz}$, minor, methine $H), 6.46-6.54(\mathrm{~m}), 6.61-6.73(\mathrm{~m}), 6.73-6.83(\mathrm{~m}), 6.86(\mathrm{~d}, J=8.2 \mathrm{~Hz})$, 
$6,92(\mathrm{dd}, J=8.0,1.7 \mathrm{~Hz}), 6.96(\mathrm{dd}, J=7.4,0.7 \mathrm{~Hz}), 7.10(\mathrm{~d}, J=7.7 \mathrm{~Hz}), 7.13-7.34(\mathrm{~m}) ;{ }^{13} \mathrm{C}$ NMR $\left(\mathrm{CDCl}_{3}\right) \delta 19.9,20.5,20.8,21.00,21.03,50.7,54.0,54.6,55.5,56.2,58.2,67.4,100.9$, 106.2, 106.6, 108.9, 112.5, 113.9, 117.8, 126.8, 127.0, 127.1, 127.4, 127.7, 127.9, 128.2, 128.7, 129.1, 129.3, 129.8, 130.3, 132.3, 132.8, 132.9, 134.9, 135.2, 135.6, 135.8, 136.0, 136.3, 138.2, 155.2, 191.7; HRMS Calcd for $\mathrm{C}_{24} \mathrm{H}_{24} \mathrm{O}_{3}: \mathrm{M}^{+}, 360.1725$. Found: $m / z 360.1726$.

\section{${ }^{1}$ H NMR integration ratio}

1.93 (s) : 1.99 (s) : 2.13 (s) : 2.15 (s) : 2.30 (s) : 3.08 (s) : 3.24 (s) : 3.72-3.83 (m) : 3.86 (s) : 3.92 (s) : 3.97-4.07 (m) : $4.53(\mathrm{dd}): 5.08(\mathrm{dd}): 6.46-6.54(\mathrm{~m}): 6.61-6.73(\mathrm{~m}): 6.73-6.83(\mathrm{~m}):$ $6.86(\mathrm{~d}): 6.92(\mathrm{dd}): 6.96(\mathrm{dd}): 7.10(\mathrm{~d}): 7.13-7.34(\mathrm{~m})=0.44: 3.21: 2.58: 0.85: 3.52: 0.36$ : $3.09: 3.34: 0.57: 0.16: 0.30: 1.00: 0.10: 0.46: 1.33: 2.22: 1.40: 1.20: 1.32: 0.21: 2.78$.

4,5-Dimethoxy-9-(2,4,6-trimethylphenyl)xanthene (3'ej). Isolated in $16 \%$ yield as a white solid; m.p. 269-276 ${ }^{\circ} \mathrm{C} ;{ }^{1} \mathrm{H}$ NMR $\left(\mathrm{CDCl}_{3}\right) \delta 1.90$ (brs, $3 \mathrm{H}$ ), 2.19 (s, $3 \mathrm{H}$ ), 2.75 (brs, 3 H), 3.59 (s, $6 \mathrm{H}), 5.80$ (s, $1 \mathrm{H}), 6.46$ (dd, $J=8.0,1.0 \mathrm{~Hz}, 2 \mathrm{H}), 6.57$ (brs, $1 \mathrm{H}), 6.67$ (dd, $J=8.2$, $1.0 \mathrm{~Hz}, 2 \mathrm{H}), 6.83$ (brs, $1 \mathrm{H}), 7.11(\mathrm{t}, J=8.2 \mathrm{~Hz}, 2 \mathrm{H}) ;{ }^{13} \mathrm{C} \mathrm{NMR}\left(\mathrm{CDCl}_{3}\right) \delta 20.7,30.9,55.4$, 105.4, 108.3, 112.5, 127.6, 134.6, 137.7, 152.3, 158.2; HRMS Calcd for $\mathrm{C}_{24} \mathrm{H}_{24} \mathrm{O}_{3}: \mathrm{M}^{+}$, 360.1725. Found: $m / z 360.1723$.

\section{Structure Determination of $\mathbf{3}$ 'ej}

The structure of $\mathbf{3}$ 'ej was determined by NOE in ${ }^{1} \mathrm{H}$ NMR. As shown below, irradiation of the methine proton of 3'ej did not enhance the $\mathrm{MeO}$ moiety, however, the absence of NOE between the methine proton and $\mathrm{H}_{\mathrm{a}}$ as well as the observed NOE in $\mathbf{3 e j}$ would support the assignment of the stereochemistry.

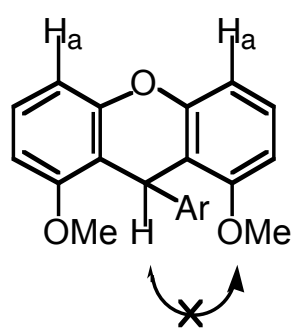

Ar: mesityl

3'ej

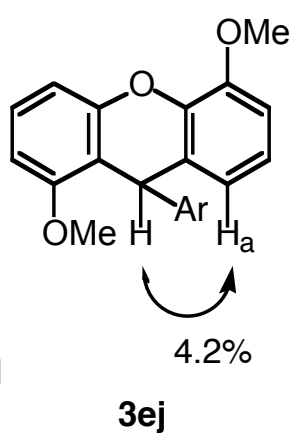

3ej

Reaction of 20 with benzyne. To a THF solution $(2.0 \mathrm{~mL})$ of $\mathbf{1 a}(0.269 \mathrm{~g}, 0.90 \mathrm{mmol}), \mathbf{2 0}$ $(0.043 \mathrm{~g}, 0.15 \mathrm{mmol})$ and 18-crown-6 (0.476 g, $1.80 \mathrm{mmol})$ was added KF (0.105 g, 1.80 $\mathrm{mmol}$ ), and the resulting mixture was stirred at $0{ }^{\circ} \mathrm{C}$ for $23 \mathrm{~h}$ before dilution with ethyl acetate. Then the mixture was filtered through a Celite plug, and concentrated. Silicagel column chromatography (hexane/ethyl acetate as an eluent) followed by gel permeation chromatography (chloroform as an eluent) gave 4,4'-dimethoxy-3,3'-di(9-xanthyl)diphenyl ether (3ao) (25\% yield) as a waxy greenish yellow solid: ${ }^{1} \mathrm{H}$ NMR $\left(\mathrm{CDCl}_{3}\right) \delta 3.74(\mathrm{~s}, 6 \mathrm{H}), 5.66(\mathrm{~s}, 2 \mathrm{H}), 6.59$ - 
6.67 (m, $4 \mathrm{H}), 6.75(\mathrm{~d}, J=9.4 \mathrm{~Hz}, 2 \mathrm{H}), 6.95(\mathrm{td}, J=7.5,1.2 \mathrm{~Hz}, 4 \mathrm{H}), 7.07$ (d, $J=8.2 \mathrm{~Hz}, 8 \mathrm{H})$, $7.17(\mathrm{td}, J=7.2,1.4 \mathrm{~Hz}, 4 \mathrm{H}) ;{ }^{13} \mathrm{C} \mathrm{NMR}\left(\mathrm{CDCl}_{3}\right) \delta 37.7,56.2,112.3,116.2,117.0,120.7$, 123.0, 124.2, 127.6, 129.3, 136.5, 151.1, 151.4, 152.3; HRMS Calcd for $\mathrm{C}_{40} \mathrm{H}_{30} \mathrm{O}_{5}: \mathrm{M}^{+}$, 590.2093. Found: $m / z$ 590.2099.

\section{References}

(1) Himeshima, Y.; Sonoda, T.; Kobayashi, H. Chem. Lett. 1983, 1211-1214.

(2) Yoshida, H.; Sugiura, S.; Kunai, A. Org. Lett. 2002, 4, 2767-2769.

(3) Peña, D.; Pérez, D.; Guitián, E.; Castedo, L. J. Am. Chem. Soc. 1999, 121, 5827-5828.

(4) Quideau, S.; Pouyse'gu, L.; Oxoby, M.; Looney, M. A. Tetrahedron 2001, 57, 319-329.

(5) Smith, J. R. L.; O’Brien, P.; Reginato, G. Tetrahedron: Asymmetry 1997, 8, 3415-3420.

(6) Kuhnert, N.; Rossignolo, G. M.; Lopez-Periago, A. Org. Biomol. Chem. 2003, 1, 1157-1170.

(7) Smith, D. W. Jr.; Babb, D. A.; Snelgrove, R. V.; Townsend, P. H. III; Martin, S. J. J. Am. Chem. Soc. 1998, 120, 9078-9079.

(8) McKinley, S. V.; Grieco, P. A.; Young, A. E.; Freedman, H. H. J. Am. Chem. Soc. 1970, 92, 5900-5907.

(9) Although 3ej has a symmetrical 9-aryl moiety (2,4,6-trimethylphenyl) in contrast to other xanthenes that form a mixture of conformers, the asymmetric center at C-9 as well as a high rotational barrier about 9 -aryl $\mathrm{sp}^{2}-\mathrm{sp}^{3}$ bond should make 3ej a mixture of conformers unless the 9-aryl moiety is perpendicular (or parallel) to the xanthyl moiety. For rotational barriers in 9-arylxanthenes, see ref. 8. 